was examined and demographic information collected. The data were divided by season - winter months incorporating October to March and summer months, April to September. The null hypothesis that there would be no difference between attendance rates in summer months compared to winter months was tested using a Mann-Whitney $U$ test. The attendance data were correlated, using Pearson's product-moment correlation coefficient, with local monthly weather data, obtained from the Met Office, for temperature, hours of sunshine and rainfall over the time period.

Results In total 506 assessment appointments were made to achieve 258 attendances, an attendance rate of $51 \%$. For assessments, there was no difference in attendance between the winter and summer months (50 and 51\% respectively). For the group PR sessions, 2325 appointments were made to achieve 1613 attendances (69\%). The overall attendance rate at group sessions during winter was $64 \%$ compared to $74 \%$ during summer. Non-parametric testing of the data revealed the seasonal difference to be statistically significant $(p<0.05)$. Attendance rates show weak, positive correlation with maximum and minimum temperatures $(r=+0.51,+0.44$ respectively) and sunlight hours $(\mathrm{r}=+0.55)$, and weak negative correlation with amount of rainfall $(\mathrm{r}=-0.33)$.

Conclusion Attendance rates were significantly worse during winter compared to summer. This needs to be taken into account when planning PR services and in local efforts to maximise patient participation. The weak correlation between attendance and specific weather indicators suggests that weather conditions may contribute to this pattern. Confounding patient factors such as illness exacerbation and environmental issues such as transport need to be further evaluated in the context of seasonality to better understand this relationship.

\section{P50 FACILITATION OF CONTINUED EXERCISE VIA PATIENT VOLUNTEERS WITH CHRONIC OBSTRUCTIVE PULMONARY DISEASE (COPD) FOLLOWING A PULMONARY REHABILITATION PROGRAMME: A FEASIBILITY STUDY}

doi:10.1136/thx.2010.150979.1

${ }^{1} \mathrm{C}$ A Langley-Johnson, ${ }^{1} \mathrm{E}$ Jenkin, ${ }^{2} \mathrm{C}$ A E Dyer, ${ }^{3} \mathrm{~K}$ Gruffydd-Jones, ${ }^{4} \mathrm{~N}$ Harris, ${ }^{5} \mathrm{M}$ Reed, ${ }^{5} \mathrm{G}$ Taylor. ${ }^{7}$ Wiltshire PCT, Chippenham, UK; ${ }^{2}$ Royal United Hospital, Bath, UK; ${ }^{3}$ Box Surgery, Chippenham, UK; ${ }^{4}$ Bath Institute of Medical Engineering, Bath, UK; ${ }^{5} B A T H$ University, Bath, UK

Introduction After pulmonary rehabilitation activity levels gradually decline, on average, back to baseline over 12-18 months. The NHS has highlighted the value of peer support for patients with chronic disease, but this has not been fully evaluated in patients with COPD. Therefore we designed an observational study with the aim of exploring and assessing an exercise maintenance programme with peer volunteer support over a 6-month period, in order to inform a future large-scale study.

Methods Patients were recruited from a 7-week outpatient community pulmonary rehabilitation programme. Six peer volunteers with COPD were trained and allocated up to six patients each. A fortnightly exercise maintenance programme was set up in one locality led by a physiotherapist \& assistant. This was supported by a programme of home-based exercise, goal setting and feedback on activity levels. Peer volunteers met with their groups each month to encourage individual activity. The primary outcomes were participation levels and attendance rates; secondary outcomes were activity levels and health status. Qualitative interviews demonstrated the individual variance in exercise habits and motivations see Abstract P50 Table 1.
Abstract P50 Table 1

\begin{tabular}{ll}
\hline Facilitators & Constraints \\
\hline Group providing social destinationSupportive & Group social activity displacing exercise \\
environment & activity \\
Group creating a shared sense of belonging & Personal outlook and life events inhibiting \\
and acceptance enhanced by common goals, & motivation and ability \\
same condition and shared characteristics & to exercise \\
Mentors adoption of counsellor/support role & Exercise programme: Lack of intensity \\
Staff generating trust through caring attitude & Self-directed, no external motivation \\
and expertise & Limited exercise equipment \\
Pre-conditioned to exercisePersonally & Fortnightly sessions \\
motivated & Group vs individual \\
Fostered through attendance at PACE & Sacrificing own needs for group cohesion \\
rehabilitation programme &
\end{tabular}

Results There were 91 potential recruits who completed rehabilitation (13 didn't meet criteria, six became unwell). 37 refused to participate, citing reasons such as poor health, no difficulty in exercising or problems travelling to the centre. Of the 35 that agreed to participate, the attendance rate at exercise was $70 \%$, and 30 completed the 6-month programme. In this group Shuttle Walking Test distances were maintained at 6 months (mean $313.2 \mathrm{~m}$ postrehabilitation compared with $317.8 \mathrm{~m}$ at 6 months, $\mathrm{p}=\mathrm{NS}$ ), and Chronic Respiratory Disease Questionnaire scores were also maintained. Qualitative analysis supported the concept of peer volunteers, while many individuals wanted flexibility and access to a range of activity programmes.

Conclusions This study suggests that maintenance programmes need to be individually tailored rather than prescriptive, in order to maximise uptake and meet the challenges posed by exacerbations. The role of peer volunteers in promoting activity is supported by the qualitative analysis, while exercise classes may help those who wish to attend.

\section{P51 GROUP-BASED PULMONARY REHABILITATION DELIVERED} TO THE HOME VIA THE INTERNET-A FEASIBILITY STUDY

doi:10.1136/thx.2010.150979.2

${ }^{1} \mathrm{D}$ J Godden, ${ }^{2} \mathrm{~J}$ Colligan, ${ }^{3} \mathrm{~A}$ Aitken, ${ }^{3} \mathrm{~A}$ Taylor. ${ }^{1}$ Centre for Rural Health, University of Aberdeen, Inverness, Scotland; ${ }^{2}$ Raigmore Hospital, Inverness, Highland, UK; ${ }^{3}$ Distance Lab, Forres, Scotland

Background Many patients cannot attend group-based pulmonary rehabilitation programmes due to remoteness from the clinic or transport problems. We describe a feasibility study of a novel method of delivering a programme via internet-based videoconferencing.

Methods We re-boxed a mini PC in a simple case with a single large on/off button, which was connected to each patient's home television and wired to the Internet, together with a web cam, speakerphone, and, where appropriate, a wireless pulse oximeter The physiotherapist delivered the programme from a central location equipped with a desktop PC connected to the internet; web cam; speakerphone and 40-in. video screen. When patients turned on their mini PC and TV and switched to the video channel, they automatically joined the class. All patients and the physiotherapist were visible and audible on all TV screens, to create a group spirit. A supporting screen was added to the physiotherapist's set-up to display patient names, exercise duration $(30-60 \mathrm{~s})$, pulse oximeter readings, and an exercise timer. A desktop video conferencing service was employed. A standard pulmonary rehabilitation exercise programme was delivered 
involving twice-weekly sessions for 8 weeks. Patients were assessed pre- and post-programme by Chronic Respiratory Questionnaire (CRQ), the Hospital Anxiety and Depression Scale (HADS) and an incremental shuttle-walking test. Satisfaction was measured using the Client Satisfaction Questionnaire (CSQ). Results are expressed as mean (SD). Four patients aged 65-79 enrolled for this feasibility study, which was approved by the North of Scotland Research Ethics Service.

Results All patients completed the programme. The technology performed well: one patient missed a single session due to a temporary problem connecting to the Internet. Clinical improvements occurred in all patients, comparable to those in a conventional programme. Compared to pre-programme values, there were improvements in: shuttle walking, 88 (35) $\mathrm{m}$; CRO dyspnoea 8 (5.2); emotion 2.8 (1); fatigue 5.3 (3.7) and mastery 4 (2.6). HADS anxiety and depression scores reduced in three of four patients. Satisfaction scores were high: CSQ values 29-32.

Conclusion The system allowed delivery of effective group-based pulmonary rehabilitation to patients in their own homes and should improve accessibility for future patients. Larger studies are warranted.

\section{P52 IS A PULMONARY REHABILITATION (PR) PROGRAMME EFFECTIVE IN REDUCING SEVERE SYMPTOMS OF ANXIETY AND DEPRESSION IN PATIENTS WITH COPD?}

doi:10.1136/thx.2010.150979.3

S Harrison, N Greening, J Williams, S Singh. Pulmonary Rehabilitation Research Group, Glenfield Hospital, University Hospitals Leicester NHS Trust, Leicester, England

Introduction Patients with COPD have a high prevalence of anxiety and depression which has been associated with poorer health outcomes. PR has been found to be successful in reducing anxiety and depression. Previous studies have not differentiated between mild, moderate and severe symptoms of anxiety and depression and therefore the efficacy of PR are unproven in treating those across a spectrum of anxious and depressed symptoms. Our study aimed to explore the effectiveness of a PR programme in reducing mild, moderate and severe symptoms of anxiety and depression in patients with COPD.

Methods Patients with a clinical and spirometric (GOLD stage $\geq 2$ ) diagnosis of COPD were identified from the PR database in Leicester, UK. Patients were categorised into three groups based on their Hospital Anxiety and Depression Scale (HADS) scores pre PR ('none' 0-7, 'probable' 8-10 and 'presence' 11-14). These patients undertook an outpatient PR programme between 2000 and January 2009 at a single centre. Changes in HADS scores were retrospectively compared. The two subscales were analysed separately.

Results 518 patients were identified (mean (SD) age 69.2 (8.80) years $\mathrm{FEV}_{1} 39.9 \%$ (15.10) predicted 310 male). Patients with a 'probable' and a 'presence' of anxiety and depression had a reduction in HADS scores pre- to post- PR $(p<0.001)$. Patients who had 'none' did not have a reduction ( $p>0.05$ ) (Abstract P52 Figure 1). An ANOVA with post hoc analysis showed a difference in the amount of change pre to post PR between the three groups. Patients with a 'presence' had the greatest reductions in their scores $(\mathrm{p}<0.05)$

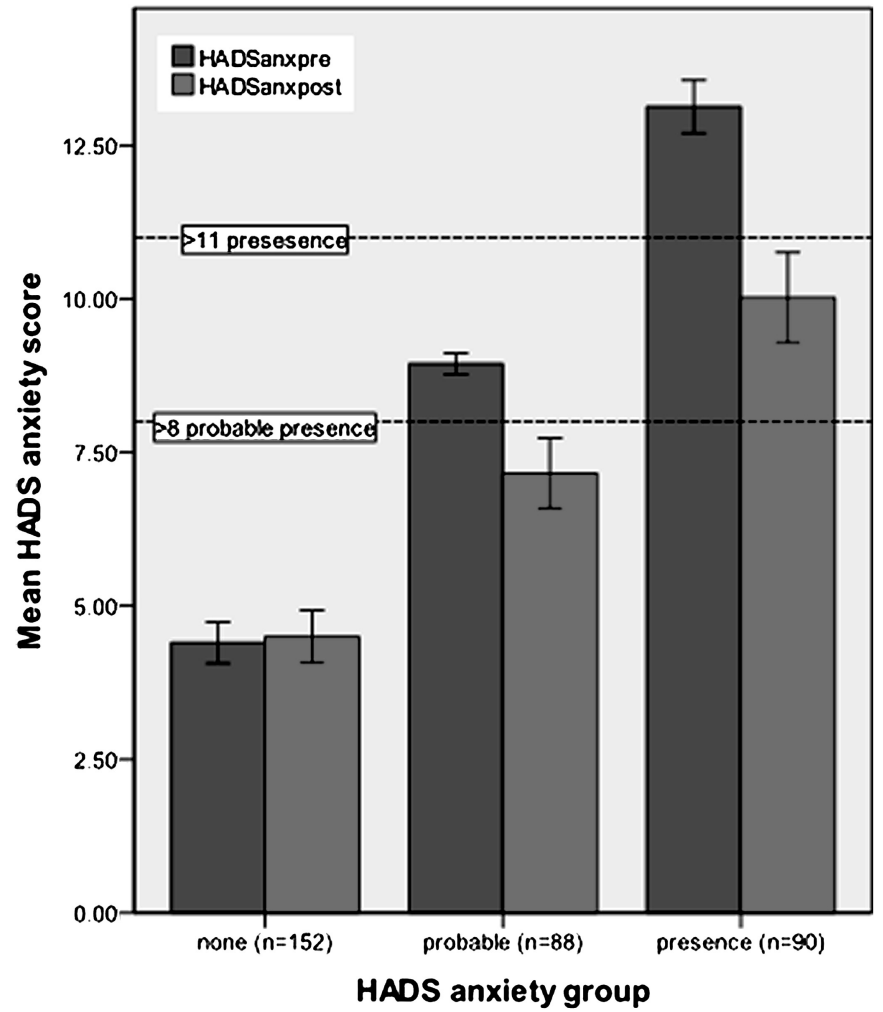

Error bars: $95 \% \mathrm{Cl}$

Abstract P52 Figure 1 Results from a paired t-test. Levels of anxiety pre and post PR as measured by the HADS.

Conclusions PR is effective in reducing moderate and severe symptoms of anxiety and depression in patients with COPD. However, $\mathrm{PR}$ is not effective in reducing severe symptoms to a level which represents no presence of anxiety and depression.

\section{P53 THE EFFECT OF CO-MORBIDITIES ON RESPONSE TO PULMONARY REHABILITATION}

doi:10.1136/thx.2010.150979.4

K A Ingram, R P Fowler, A L Clark, C M Nolan, W D Man. Harefield Pulmonary Rehabilitation Team and Biomedical Research Unit, Royal Brompton \& Harefield NHS Foundation Trust, Harefield, UK

Introduction Most patients with COPD are elderly, and the presence of co-morbidities is not uncommon. Co-morbidities are an important determinant of health related quality of life in patients with COPD. Although pulmonary rehabilitation (PR) is well known to improve exercise performance, health-related quality of life and symptoms, a proportion of patients are non-responders. We hypothesised that improvement in exercise capacity and healthrelated quality of life following PR may be limited in the presence of co-morbidities.

Methods A structured history was taken from 128 patients referred to an 8-week supervised outpatient PR programme. Individuals' selfreported co-morbidities were assessed by the Charlson index, which assigns to each disease a score that is proportional to the disease related risk of death. The calculated Charlson index did not include COPD in the individual's score as suggested in the original description. In 115 patients completing the programme, changes in incremental shuttle walk (ISW) and chronic respiratory disease questionnaire (CRQ) domains following PR were calculated, and 